\title{
Respiratory Manifestations, Radiologic Findings and Respiratory Management of Hospitalized Children with COVID-19 among Different Age Groups: A Systematic Review
}

\author{
Maria Cristina H. Lozada, MD, ${ }^{1}$ Fides Roxanne M. Castor, MD² and Kevin L. Bautista, MD ${ }^{1}$ \\ ${ }^{1}$ Division of Pediatric Pulmonology, Department of Pediatrics, Philippine General Hospital, University of the Philippines Manila \\ ${ }^{2}$ Division of Pediatric Emergency Medicine, Department of Pediatrics, Philippine General Hospital, University of the Philippines Manila
}

\begin{abstract}
Objective. This paper aims to determine the respiratory manifestations, radiologic findings, and management of coronavirus-19 (COVID-19) among the hospitalized children of different age groups based on a systematic review of literature.

Methodology. We searched the MEDLINE, Google Scholar, Cochrane, and MedRvrix databases for relevant studies on pediatric COVID-19 done between January 1 to May 25, 2020. Data on demographic features, pulmonary symptoms, chest imaging findings, and oxygen support were extracted in a data abstraction form and analyzed among the different pediatric age groups.
\end{abstract}

Results. Sixteen studies were included in this systematic review, with a total of 61 patients. Thirty-nine patients (64\%) belong to the 2 to 12 year age group. Cough was the most commonly reported respiratory symptoms among children older than one month. Most of the patients, particularly adolescents, had asymptomatic or mild infection. On chest imaging, infiltrates and ground-glass opacities were the most common abnormalities. Oxygen supplementation via nasal cannula, non-invasive ventilation, and mechanical ventilation was noted in three cases.

Conclusion. Our systematic review shows that COVID-19 can present with a spectrum of respiratory manifestations and disease severity as well as a variety of radiologic abnormalities across different pediatric age groups. While most children do not develop hypoxemia warranting oxygen supplementation, a severe respiratory infection can still manifest in children. Asymptomatic and milder infections are common and highlight the need for appropriate respiratory precautions. More pediatric studies on the pulmonary manifestations and management of children with COVID-19 can aid in a better understanding of this novel disease.

Key Words: COVID-19, pediatric, respiratory, management

\section{INTRODUCTION}

Coronavirus Disease 19 (COVID-19) is a global health concern with a spectrum of clinical manifestations ranging from mild to critical. ${ }^{1}$ With an increasing number of cases of COVID-19 reported in different locations, ${ }^{2-3}$ the respiratory manifestations and management of COVID-19 among pediatric patients of other age groups should be reviewed. COVID-19 may present with mild

Corresponding author: Maria Cristina H. Lozada, MD Division of Pediatric Pulmonology

Department of Pediatrics

Philippine General Hospital

University of the Philippines Manila

Taft Avenue, Manila, Philippines

Email:mhlozada@up.edu.ph illness in children or may be rare in neonates and infants, but pediatric patients are still vulnerable to this highly infectious disease..$^{4-5}$ Presently, there are few studies on the respiratory manifestations and management of COVID-19 in hospitalized children of varying age groups. It is important 
to examine data on the respiratory manifestations and radiologic presentation of COVID-19 in children, as these may impact the treatment strategies and patient outcomes of pediatric COVID-19.

This systematic review aimed to describe the respiratory manifestations, radiologic findings, and respiratory management strategies for COVID-19 among different age groups of laboratory-confirmed COVID-19 hospitalized pediatric patients, based on exhaustive search, appraisal, and synthesis of existing published data on this subject.

\section{Specifically, we aimed}

1. To determine the presenting respiratory manifestations and disease severity of hospitalized children of different age groups ( $0-1$ month, 1 month- 2 years, $2-12$ years, and 12-18 years) with laboratory-confirmed (based on reverse transcriptase polymerase chain reaction) COVID-19;

2. To identify the radiologic findings (chest $x$-ray, chest ultrasound, and chest computed tomography scan) among the different age groups of hospitalized children with COVID-19; and

3. To review the emergent or initial pulmonary interventions given (oxygen support, assisted ventilation, and/or bronchodilator administration) to hospitalized children with COVID-19.

\section{METHODS}

We followed the Preferred Reporting Items for Systematic Reviews and Meta-Analyses (PRISMA) ${ }^{6}$ to answer our research question "What are the respiratory manifestations, radiologic findings and respiratory management of COVID-19 among the hospitalized children of different age groups?”

\section{Literature Search}

\section{Search Strategy}

An exhaustive literature search was conducted to identify relevant studies on the respiratory manifestations, radiologic findings, and respiratory treatment for COVID-19 among different age groups of hospitalized children with this condition.

The following scientific databases were utilized in the literature search: MEDLINE, Google Scholar, Cochrane, and Clinicaltrials.gov. Preprint articles were also searched from the MedRvrix database. The following Medical Subject Headings (MeSH) terms were used in the search: "COVID-19", "respiratory", "manifestations", "management", "children", "neonates", "infants", and "adolescents".

\section{Study Selection}

The literature search was restricted to studies published from January 1, 2020, to May 25, 2020. Among studies that fulfilled the inclusion criteria, all types of study designs were reviewed. The inclusion criteria were as follows: (1) studies involving hospitalized COVID-19 pediatric patients 0-18 years old; (2) studies including laboratory-confirmed COVID-19 disease by reverse-transcription polymerase chain reaction; and (3) studies detailing the respiratory features, radiologic test results, and/or pulmonary management strategies of COVID-19 infection.

We excluded reviews, commentaries, editorials, studies exclusively done on adult populations, and articles not written in English.

\section{Outcome Measures}

Our primary outcome of interest in this review is the respiratory manifestations and management (specifically oxygen support, non-invasive ventilatory support, assisted ventilation, or bronchodilator treatment) of laboratoryconfirmed and hospitalized COVID-19 children of varying age groups. The other outcomes of this review include the following: (1) demographic profiles, (2) chest imaging findings, (3) baseline oxygenation status, and (4) outcomes (survival, death, or still admitted) of pediatric patients with COVID-19.

\section{Abstract Screening and Data Extraction}

\section{Title and Abstract Screening}

All retrieved titles of studies fulfilling the aforementioned inclusion criteria were screened. Duplicate papers were removed. The remaining articles were screened based on the title and abstract; studies not related to the topic or papers which do not address the population, intervention, comparison, and outcome (PICO) for our study were excluded.

Two authors (MCL and FRC) independently reviewed each abstract for eligibility using a screening form. Any differences in the independent review results were discussed, and a consensus was reached between the reviewers.

\section{Data Abstraction}

After screening eligible abstracts, two authors (MCL and FRC) independently reviewed each full-text article and recorded data from included studies into a standardized data abstraction form. The data extraction form was designed and was pilot tested by the authors. The said form contains the following for each included study: title of study, principal investigator or first author, date of publication, type of study, study setting, number of participants, ages and range, respiratory and non-respiratory clinical manifestations, demographic profiles, disease severity classification, radiologic test results as available (chest $\mathrm{x}$-ray, ultrasound or computed tomography scan), oxygenation status (based on peripheral oxygen saturation and arterial blood gas parameters), initial respiratory treatment given (oxygen support, assisted ventilation, bronchodilators) and outcomes (mortality, survival or still admitted). Included studies were critically appraised, and relevant data were synthesized. 


\section{Quality Assessment and Data synthesis Plan}

The Quality Assessment Tool for Case Series Studies was utilized to assess study quality ${ }^{7}$ among case series studies. Descriptive statistics were used to describe continuous variables (means, frequency, and proportions).

\section{Ethical Considerations}

The research protocol was submitted to the UP Manila Research Ethics Board (UPMREB) before the commencement of the study and was granted exemption from ethical review (UPMREB 2020-499-EX).

\section{RESULTS}

\section{Summary of Studies and Demographic Profiles of Included Patients}

A total of 16 studies $^{8-23}$ were included in this systematic review; the PRISMA diagram is shown in Figure 1. The included studies'study designs were two retrospective analyses, 7 case reports, and 7 case series. There were nine studies done in China ${ }^{8-16}, 1$ in Switzerland ${ }^{17}, 1$ in the United States ${ }^{18}, 2$ in Italy ${ }^{19-20}$, and 1 study each from Korea ${ }^{21}$, Belgium ${ }^{22}$ and Lebanon. ${ }^{23}$

There were a total of 61 hospitalized patients with positive SARS-CoV-2 RT-PCR results, with an age range of 15 days to 16 years. Thirty-five (57\%) of the pediatric patients included were males. No mortalities were noted in $13 / 16$ studies that reported clinical outcomes of patients. The summary of the included studies is shown in Table 1.

\section{Respiratory Manifestations and Disease Severity of COVID-19 among the different pediatric age groups}

All the sixteen included studies in our review reported the clinical manifestations and ages of the pediatric patients admitted for COVID-19. Most of the children admitted for COVID-19 belong to the 2 to 12 year age group (39/61). Cough was the most common respiratory symptom reported in the 1 month - 2 year (70\%), 2-12 year (15\%), and 1218 year $(36 \%)$ age groups. Fever was the most common non-respiratory symptom of COVID-19 in the same age brackets. On the other hand, among neonates, tachypnea and desaturation were observed in one patient with concomitant cyanotic heart disease. ${ }^{16}$ At the same time, poor oral intake $^{18}$ and asymptomatic infection ${ }^{22}$ were also reported in this age group (Table 2).

Among eleven papers (with a total of 40 patients) which reported disease severity ${ }^{8-11,13-15,17,18,21,22}$, most of the patients were classified as having asymptomatic infection or mild or ordinary disease severity (Table 3 ). The study by Dallan et al. reported a ten-year-old patient with SARSCoV-2 RT-PCR confirmed severe infection and shock who developed Multi-system Inflammatory Syndrome in Children temporally associated with COVID-19 (MIS-C). ${ }^{17}$ A 15-day old neonate with Tetralogy of Fallot also developed

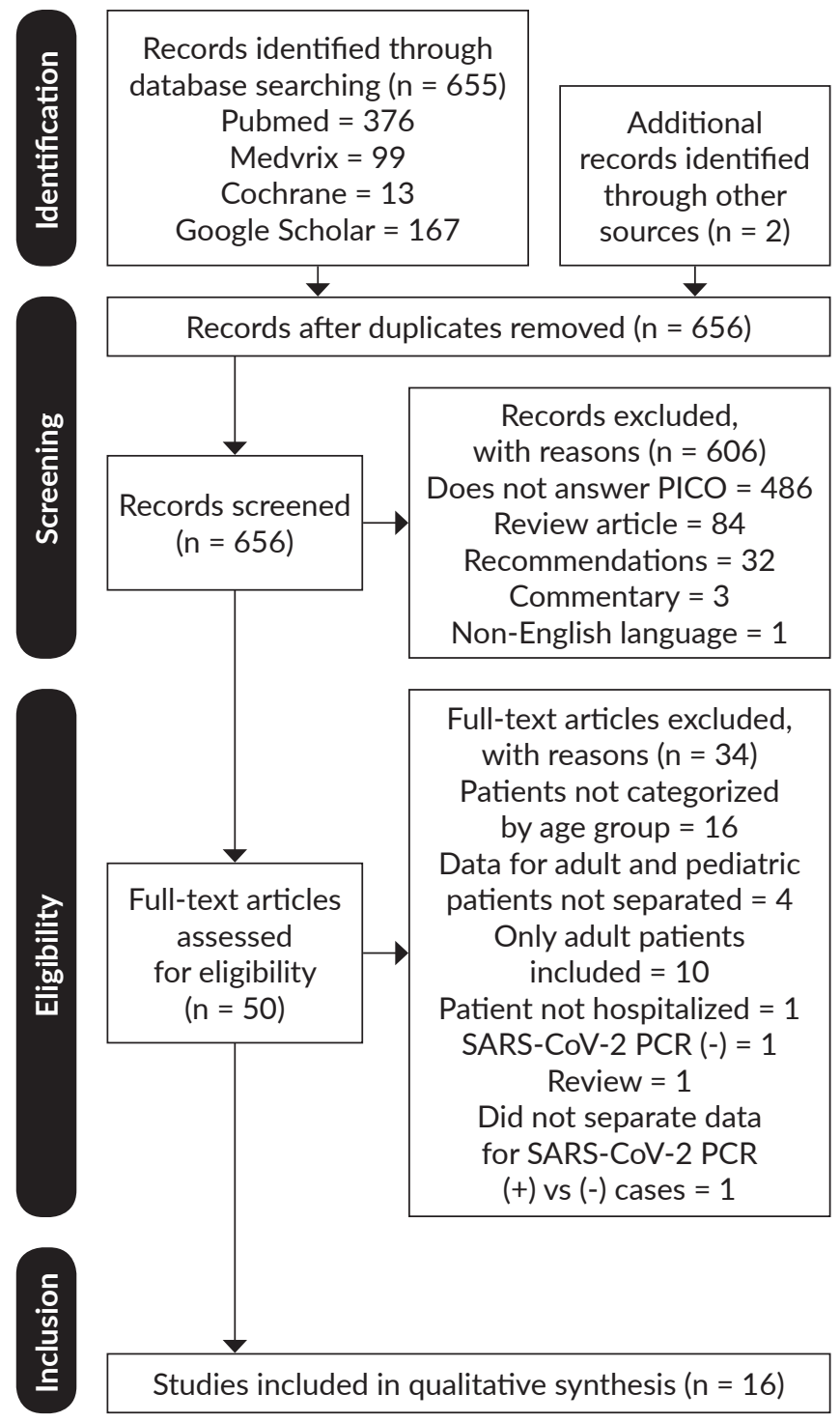

Figure 1. The PRISMA Diagram.

COVID-19 and was intubated as reported in the paper by Salik $^{16}$ et al. In contrast, Cui et al. reported a 55 -day-old with severe COVID-19 who was placed on oxygen support via nasal cannula. ${ }^{11}$

\section{Chest Imaging Findings of COVID-19 among the different pediatric age groups}

Chest $\mathrm{x}$-ray of pediatric patients with COVID-19 were reported in 7 studies $12,13,16,17,20,22,23$ (total of 18 patients), while chest CT scan findings were indicated in 9 papers ${ }^{8-15,21}$ (total of 37 patients). On chest $x$-ray, bilateral infiltrates were reported in two neonates. ${ }^{16,22}$ In the 2-12 year age group, bronchovascular shadows and normal findings were the most common radiograph findings. Three out of five adolescents with chest $\mathrm{x}$-ray done had unremarkable results (Table 4).

On the other hand, on chest CT scan, ground-glass opacities in the lower lobes were observed in most cases. 
Table 1. Summary of Included Studies

\begin{tabular}{|c|c|c|c|c|c|c|c|c|}
\hline $\begin{array}{l}\text { No. of } \\
\text { study }\end{array}$ & $\begin{array}{l}\text { First } \\
\text { Author }\end{array}$ & Study Site & $\begin{array}{l}\text { Number of SARS- } \\
\text { CoV-2 positive } \\
\text { pediatric patients }\end{array}$ & Study design & Age/ Range & Males & $\begin{array}{l}\text { Co-morbid } \\
\text { conditions }\end{array}$ & Clinical manifestations \\
\hline 1 & Zhang8 & Tianjun, China & 3 & Case series & $6-9$ years & $3 / 3$ & Not reported & $\begin{array}{c}\text { Cough, nasal congestion, } \\
\text { sore throat }\end{array}$ \\
\hline 2 & Song ${ }^{9}$ & Hubei, China & 16 & $\begin{array}{l}\text { Retrospective } \\
\text { analysis }\end{array}$ & $\begin{array}{l}11 \text { months - } \\
14 \text { years }\end{array}$ & $10 / 16$ & Not reported & Asymptomatic, fever, cough \\
\hline 3 & $\operatorname{Tan}^{10}$ & $\begin{array}{c}\text { Hubei and } \\
\text { Changsa, China }\end{array}$ & 10 & $\begin{array}{l}\text { Retrospective } \\
\text { analysis }\end{array}$ & 1-7 years & $3 / 10$ & None & $\begin{array}{l}\text { Asymptomatic, fever, } \\
\text { abdominal pain, vomiting, } \\
\text { constipation, convulsions }\end{array}$ \\
\hline 4 & Cui ${ }^{11}$ & Guiyang, China & 1 & Case report & 55 days & $0 / 1$ & None & $\begin{array}{c}\text { Cough, nasal } \\
\text { congestion, dyspnea }\end{array}$ \\
\hline 5 & $\mathrm{Lu}^{12}$ & $\begin{array}{l}\text { Guangzhou, } \\
\text { China }\end{array}$ & 9 & Case series & $\begin{array}{l}2 \text { months - } \\
15 \text { years }\end{array}$ & $5 / 9$ & Not reported & $\begin{array}{c}\text { Cough, nasal } \\
\text { congestion, fever }\end{array}$ \\
\hline 6 & Yin $^{13}$ & Beijing, China & 1 & Case report & 9 years & $1 / 1$ & Not reported & Fever \\
\hline 7 & Chan $^{14}$ & Wuhan, China & $1^{*}$ & Case series & 10 years & $1 / 1$ & None & None \\
\hline 8 & $\mathrm{Xu}^{15}$ & Guizhou, China & $2 *$ & Case series & 10 years & $1 / 2$ & Not reported & None \\
\hline 9 & Salik ${ }^{16}$ & Wuhan, China & 1 & Case report & 15 days & $0 / 1$ & Tetralogy of Fallot & $\begin{array}{l}\text { Desaturation, tachypnea, } \\
\text { cyanosis, feeding } \\
\text { intolerance, lethargy }\end{array}$ \\
\hline 10 & Dallan ${ }^{17}$ & $\begin{array}{l}\text { Geneva, } \\
\text { Switzerland }\end{array}$ & $1^{* *}$ & Case series & 12 years & $1 / 1$ & Obesity, Asthma & $\begin{array}{c}\text { Cough, dyspnea, } \\
\text { odynophagia, headache, } \\
\text { hypoventilation, fever }\end{array}$ \\
\hline 11 & Dumpa $^{18}$ & New York, US & 1 & Case Report & 22 days & $1 / 1$ & None & Fever, decreased oral intake \\
\hline 12 & Musolino $^{19}$ & Rome, Italy & 10 & Case series & $1-16$ years & $8 / 10$ & None & $\begin{array}{l}\text { Cough, fever, headache, } \\
\text { chest pain, arthralgia }\end{array}$ \\
\hline 13 & Verdoni ${ }^{20}$ & Bergamo, Italy & 2 & Case series & $7-7.5$ years & $1 / 2$ & $\begin{array}{c}\text { Congenital adrenal } \\
\text { hyperplasia (1) }\end{array}$ & Diarrhea, meningeal signs \\
\hline 14 & Park $^{21}$ & Korea & 1 & Case report & 10 years & $0 / 1$ & Not reported & Fever \\
\hline 15 & Piersigilli22 & $\begin{array}{l}\text { Brussels, } \\
\text { Belgium }\end{array}$ & 1 & Case report & 26 days & $1 / 1$ & $\begin{array}{c}\text { Prematurity, Patent } \\
\text { Ductus arteriosus }\end{array}$ & No symptoms \\
\hline 16 & Mansour $^{23}$ & Lebanon & 1 & Case report & $\begin{array}{l}1 \text { year } 4 \\
\text { months }\end{array}$ & $0 / 1$ & None & Fever, diarrhea, rhonchi \\
\hline
\end{tabular}

Abbreviations: $C T$ = computed tomography, UTZ = ultrasound, GGO = ground glass opacities

Note: * the pediatric patients were part of a family cluster **only one pediatric patient in this series had positive RT-PCR results for SARS-CoV-2

Ground glass opacities in the subpleural and upper and lower lobes were the most commonly reported chest CT scan abnormalities in the adolescent age group. The paper by Musolino et al. reported chest ultrasound findings of 10 patients; pleural irregularities and vertical artifacts were the most commonly reported findings. ${ }^{19}$ The rest of the imaging findings of COVID-19 across the different age groups of children are listed in Table 4.

\section{Respiratory Management of COVID-19 patients}

Only three children in our review were found to be hypoxemic and warranted oxygen support. The paper by Salik et al. reported a 15-day old infant with Tetralogy of Fallot who had cyanosis, desaturation, and apneic episodes and was subsequently intubated. ${ }^{16} \mathrm{~A}$ young infant presented with cough and dyspnea was placed on oxygen support via nasal cannula as reported by Cui et al. ${ }^{11}$. In contrast, Piersigilli et al. reported a 26-day preterm baby placed on non-invasive ventilation and was later shifted to continuous positive airway pressure (CPAP). ${ }^{22}$

\section{DISCUSSION}

\section{Summary of Main Findings}

Our systematic review yielded sixteen studies (a total of 61 cases) on the respiratory manifestations, radiologic findings, and respiratory management of hospitalized children with laboratory-confirmed COVID-19. Thirtynine patients $(64 \%)$ belong to the 2 to 12 -year age-group. Most of the patients had a mild disease or asymptomatic 
CT scan: GGO (2/3), new cord shadow $(1 / 3)$

Chest CT scan: normal, groundglass opacities, patchy shadows, consolidation

Chest CT scan: normal, mottling, bronchopneumonia-like changes, ground-glass opacities

Chest CT scan: patchy groundglass opacity

Chest $\mathrm{x}$-ray: bronchovascular shadows, hilar shadows

Chest CT scan: GGO

Chest x-ray: normal
Chest CT scan: cord shadow

Chest CT scan: GGOs

Chest CT scan: Normal

Chest $x$-ray: bilateral opacities, reduced lung volumes
Not reported

Not reported

Not reported Oxygen support via nasal cannula, interferon alpha nebulization Not reported None

Not reported

\section{None}

Invasive mechanical ventilation

None

Crystalloid bolus, trial of salbutamol

Antibiotics, interferon nebulization,

Lopinavir-ritonavir, azithromycin,
traditional Chinese medicine

Bed rest, symptomatic treatment Not reported

Oral catillan, Iysine acetylsalicylate injection

Not reported

Surgery for Tetralogy of Allot ribavirin, vitamin $\mathrm{C}$, Chinese oral medicine oseltamivir, arditol, cefamandole, reduced glutathione, ursodeoxycholic acid

Chest $\mathrm{x}$-ray: Normal

No oxygen supplementation

Antibiotics

None

Not reported

Not reported

Inhaled interferon, lopinavir/ritonavir

Not reported

Discharged (1/1) irregularities, vertical artifacts

Chest $\mathrm{x}$-ray: pneumonia

Not reported

Not reported

None

Discharged (1/1)

Chest CT scan: consolidation with GGO

Chest $x$-ray: bilateral infiltrates

Chest $x$-ray: consolidation, infiltrates
No oxygen supplementation

Non-invasive ventilation shifted to continuous positive airway pressure

No oxygen supplementation
Ceftriaxone, Metronidazole
Still admitted

Discharged (1/1)

Table 2. Respiratory Manifestations of COVID-19 among the different pediatric age groups

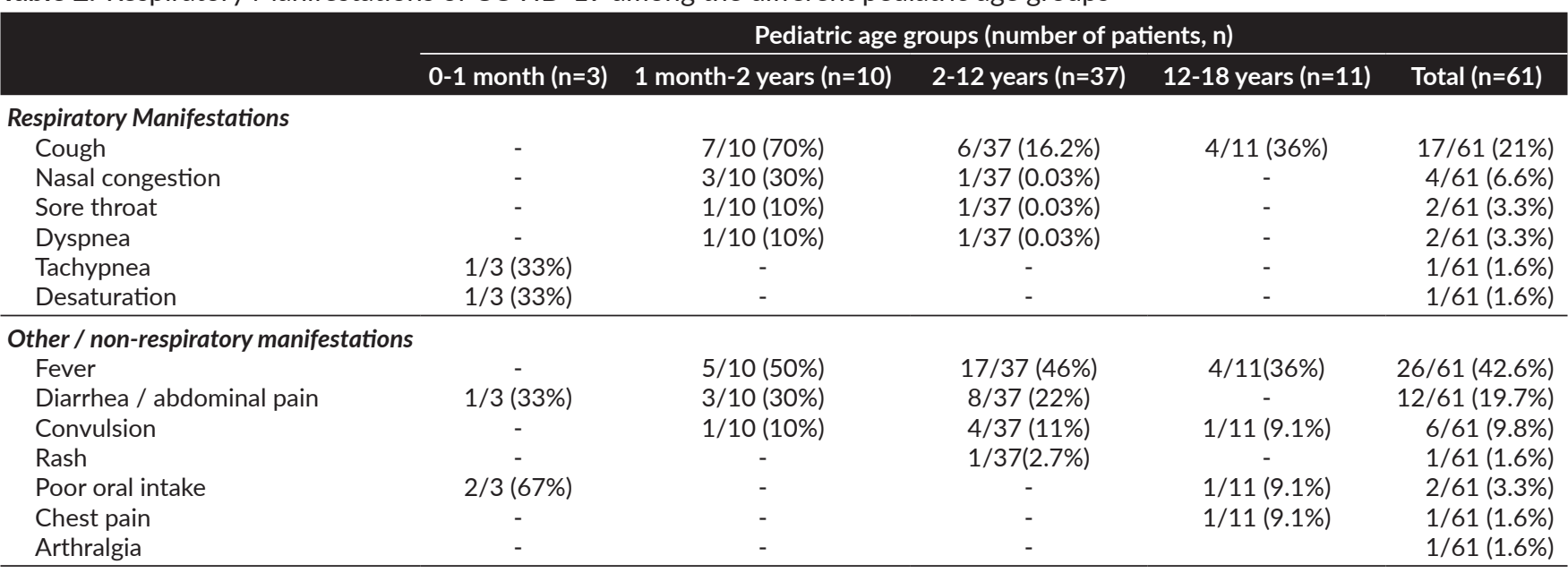


Table 3. Disease Severity of COVID-19 among the different pediatric age groups

\begin{tabular}{lcccc} 
& \multicolumn{4}{c}{ Pediatric age groups (number of patients, $\mathbf{n})$} \\
\cline { 2 - 5 } & $\mathbf{0 - 1}$ month $(\mathbf{n}=\mathbf{3})$ & $\mathbf{1}$ month-2 years $(\mathbf{n}=\mathbf{5})$ & $\mathbf{2 - 1 2}$ years $(\mathbf{n}=\mathbf{2 4})$ & $\mathbf{1 2 - 1 8}$ years $(\mathbf{n}=\mathbf{8})$ \\
\hline Asymptomatic & $1 / 3(33 \%)$ & $1 / 5(20 \%)$ & $6 / 24(25 \%)$ & $7 / 8(86 \%)$ \\
Mild / Ordinary & $1 / 3(33 \%)$ & $3 / 5(60 \%)$ & $17 / 24(71 \%)$ & $1 / 8(14 \%)$ \\
Severe & - & $1 / 5(20 \%)$ & $1 / 24(4.1 \%)$ & - \\
Critical & $1 / 3(33 \%)$ & - & - & - \\
\hline
\end{tabular}

Table 4. Chest Imaging Findings of COVID-19 among the different pediatric age groups

\begin{tabular}{|c|c|c|c|c|}
\hline \multirow{2}{*}{ Imaging Test and Findings } & \multicolumn{4}{|c|}{ Pediatric age groups (number of patients, $n$ ) } \\
\hline & 0-1 month & 1 month- 2 years & 2-12 years & $12-18$ years \\
\hline \multicolumn{5}{|l|}{ Chest x-ray } \\
\hline Bilateral infiltrates & $2 / 2$ & $1 / 3$ & $1 / 8$ & $1 / 5$ \\
\hline Lobar Consolidation & - & $1 / 3$ & - & - \\
\hline Unilateral interstitial infiltrates & - & - & - & $1 / 5$ \\
\hline Infiltrates, location unspecified & - & - & $1 / 8$ & - \\
\hline Bronchovascular shadows & - & $1 / 3$ & $3 / 8$ & - \\
\hline Decreased lung volume & $1 / 8$ & - & - & - \\
\hline Normal & - & $1 / 3$ & $3 / 8$ & $3 / 5$ \\
\hline \multicolumn{5}{|l|}{ Chest CT-scan } \\
\hline Subpleural or peripheral Ground glass opacities & - & $1 / 6$ & $3 / 21$ & $2 / 10$ \\
\hline GGO, upper lobe & - & - & - & $3 / 10$ \\
\hline GGO, lower lobe & - & - & $7 / 21$ & $3 / 10$ \\
\hline GGO, upper and lower lobe & - & $1 / 6$ & $1 / 21$ & - \\
\hline Bronchial wall thickening, increased lung markings & - & $1 / 6$ & - & - \\
\hline Nodular shadow & - & - & $2 / 21$ & $1 / 10$ \\
\hline Patchy shadow & - & $1 / 6$ & $1 / 21$ & $1 / 10$ \\
\hline Unilateral GGO, location unspecified & - & - & - & - \\
\hline New cord shadow & - & - & $2 / 21$ & - \\
\hline Bilateral infiltrates & - & - & $1 / 21$ & $1 / 10$ \\
\hline Consolidation & - & - & $1 / 21$ & $1 / 10$ \\
\hline Parenchymal bands & - & - & - & - \\
\hline "Halo sign" & - & - & $1 / 21$ & $5 / 10$ \\
\hline Normal & - & $3 / 6$ & $6 / 21$ & \\
\hline \multicolumn{5}{|l|}{ Chest ultrasound } \\
\hline Unilateral pleural irregularities & - & - & - & $2 / 5$ \\
\hline Bilateral pleural irregularities & - & - & $2 / 3$ & $2 / 5$ \\
\hline Vertical artifacts & - & $2 / 2$ & $1 / 3$ & $2 / 5$ \\
\hline Unilateral Consolidation & - & - & - & $1 / 5$ \\
\hline
\end{tabular}

infection, but the severe infection was identified in two cases and critical infection in one patient.

Cough was the most commonly reported respiratory manifestation among children with COVID-19, while fever and gastrointestinal symptoms were frequently observed. On chest imaging, a wide spectrum of findings was noted. While some patients had normal chest imaging findings, infiltrates and ground-glass opacities were the most commonly observed abnormalities. Most patients did not require oxygen supplementation —only three papers reported using oxygen supplementation via nasal cannula, non-invasive ventilation, and mechanical ventilation.

\section{Respiratory Manifestations of COVID-19}

The WHO has classified the severity of pediatric COVID-19 based on clinical features and hypoxemia. ${ }^{1}$ Several papers have reported that most children with COVID-19 were asymptomatic or of milder severity. ${ }^{24-27}$ Among the possible reasons for milder disease observed in children include less virus exposure, innate immunity, or lower affinity of angiotensin-converting-enzyme 2 (ACE2) receptor in children. ${ }^{9,21,28}$

One review revealed that infants were observed to have a higher proportion of severe and critical illness severity. ${ }^{26}$ Results of our study also showed similar findings. Among studies that reported disease severity, while most patients either had an asymptomatic or mild infection, it is worth noting that there were still cases of severe and critical disease. A more significant proportion of children with COVID-19 were observed to exhibit fever in our review, but cough remained the most common presenting respiratory complaint (Table 3). Similarly, based on the papers by Yasahura et al. ${ }^{26}$ and Christophers et al. ${ }^{27}$ most pediatric patients with COVID-19 were identified to be either asymptomatic or have a single and non-respiratory symptom at initial presentation. ${ }^{26-27}$

On the other end of the spectrum, young infants can manifest with rapid progression of an illness, as reported by 
Cui et al. ${ }^{11}$ Based on the American Academy of Pediatrics interim guidelines, a multi-system inflammatory syndrome in children (MIS-C) should be considered among children with severe COVID illness, multiple organ involvement and laboratory parameters reflecting marked inflammation. ${ }^{29}$ The said guidelines also state that the respiratory manifestations seen in adults may not be present among children. ${ }^{29}$ Dallan et al. reported a 10 -year-old child presenting initially with tachypnea, tachycardia, hypoventilation, and wheezing and developed compensated shock. ${ }^{17}$ The child was diagnosed with MIS-C, and the authors emphasize that clinicians should be wary that critical illness may develop among children with COVID-19. ${ }^{17}$ Thus, even with a paucity of respiratory symptoms, children with MIS-C warrant closer monitoring and a higher level of care. Furthermore, a recent paper by Parisi et al., published in December 2020, reported that Acute Respiratory Distress Syndrome (ARDS) might develop after the first week of COVID-19 illness. This may manifest with progressive and rapid deterioration among children. ${ }^{30}$

\section{Radiologic Manifestations of COVID-19}

Foust and colleagues' comprehensive paper presented recommendations for describing COVID-19 chest imaging findings as typical, indeterminate, atypical, and negative. ${ }^{31}$ Nino et al. reported that chest imaging results of children with COVID-19 were less common and of lesser severity than adult patients; around one-third may have normal chest CT scan findings. ${ }^{32}$ In our review, we found that several papers reported a variety of chest $\mathrm{x}$-ray and chest CT scan abnormalities of laboratory-confirmed COVID-19. These may include ground-glass opacities, consolidation, patchy changes, and infiltrates $8,10-14,16,21,22$ while normal

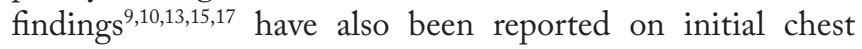
imaging. Typical findings for pediatric COVID-19, which may serve as helpful diagnostic aids and triaging,include ground-glass opacities and/or consolidation distributed bilaterally in the peripheral and subpleural regions. ${ }^{31,33}$

\section{Respiratory Management of COVID-19}

Most of the papers in our review yielded cases of generally milder severity; only three cases were identified to need respiratory support, given via nasal cannula ${ }^{11}$, noninvasive ventilation ${ }^{22}$, and invasive mechanical ventilation. ${ }^{16}$ Based on the WHO guidelines ${ }^{1}$ and Pediatric Intensive Care Unit guidelines ${ }^{32}$ on the management of COVID-19, among hypoxemic children with COVID, oxygen support can initially start with a low-flow nasal cannula. It can be escalated to a non-rebreathing face mask, high-flow nasal cannula, or non-invasive ventilation if hypoxemia is persistent. The patient's vital signs and acid-base status should be monitored closely to detect clinical deterioration. ${ }^{30}$ Hypoxemic respiratory failure for patients already tried on less invasive modes of oxygen support may prompt the need for endotracheal intubation and mechanical ventilation. ${ }^{1,34}$
Presently, the mechanism of transmission of the SARSCoV-2 is believed to be via droplets and direct contact. ${ }^{35}$ The possibility of aerosol transmission is also considered. ${ }^{35}$ A recent paper reports that children of all ages may have high nasopharyngeal viral loads of SARS-CoV-2, especially during the first two days of symptom onset. ${ }^{36}$ Precautions including appropriate personal protective equipment and use of negative pressure rooms is recommended, especially during respiratory procedures and in modes of oxygen or ventilator support that may be potentially aerosol-generating, such as high-flow oxygen, non-invasive ventilation, or intubation. . $24,32,35^{2}$

\section{Limitations and Recommendations}

The relatively small number of papers limits this review. Our principal outcomes of interest (respiratory manifestations, radiologic findings, and pulmonary management) are categorized and described individually or according to the set patient age group. Furthermore, we only included studies conducted among hospitalized children with SARSCoV-2 RT-PCR confirmed infection; patients managed as probable COVID-19 or those in the outpatient or community facility settings were not included. Lastly, most of the studies included in the final analysis were confined to particular geographic locations. They may not represent the clinical presentation of pediatric COVID-19 in other areas of the world.

Thus, more studies, particularly prospective studies, designed to analyze the respiratory manifestations, management, and outcomes of COVID-19 in children are recommended. Other aspects that should be explored are the objective measures of respiratory impairment (such as peripheral oxygen saturation) and its correlation with the severity of COVID-19 and outcomes in children who develop more severe disease and hypoxemia.

\section{CONCLUSION}

Among different pediatric age groups, COVID-19 can present with a spectrum of respiratory manifestations and disease severity and a variety of radiologic abnormalities. Our systematic review shows that cough was the most commonly reported respiratory symptoms among children older than one month. Most of the patients, particularly adolescents, had asymptomatic or mild infection. Among children with chest imaging done, ground-glass opacities were the most commonly observed abnormalities; however, normal chest imaging has been reported in children with COVID-19.

Furthermore, while most children do not develop hypoxemia warranting oxygen supplementation, a severe respiratory infection can still manifest in children. More pediatric studies on the pulmonary manifestations and management of children with COVID-19 can aid in a better understanding of this novel disease and potentially improve patient outcomes. 


\section{Statement of Authorship}

The final paper has been approved for submission by all authors.

\section{Author Disclosure}

The authors declared no conflicts of interest.

\section{Funding Source}

None.

\section{REFERENCES}

1. World Health Organization. Clinical management of COVID-19 Interim Guidance. May 2020. [Internet]. [cited 2020 Nov 22]. Available from: https:/www.who.int/publications/i/item/clinicalmanagement-of-covid-19.

2. Lai CC, Liu YH, Wang CY, Wang YH, Hseuh SC, Yen MY, et al. Asymptomatic carrier state, acute respiratory disease, and pneumonia due to severe acute coronavirus 2 (SARS-CoV-2): Facts and myths. Journal of Microbiology, Immunology and Infection. J Microbiol Immunol Infect 2020 Jun;53(3):404-412.

3. Cheng ZJ, Shan J. 2019 Novel coronavirus: where we are and what we know. Infection 2020; 48:155-16.

4. She J, Liu L, Liu W. COVID-19 epidemic: Disease characteristics in children. J Med Virol. 2020;1-8.

5. Hong H, Wang Y, Chung HT, Chen CJ. Clinical characteristics of novel coronavirus disease 2019 (COVID-19) in newborns, infants and children. Pediatrics and Neonatology 2020; 61:131-2.

6. Moher D, Liberati A, Tetzlaff J, Altman DG, The PRISMA Group (2009). Preferred Reporting Items for Systematic Reviews and MetaAnalyses: The PRISMA Statement.PLoS Med 6(7): e1000097.

7. Quality Assessment Tool for Case Series Studies. [Internet]. [cited 2020 Nov 22]. Available from https://www.nhlbi.nih.gov/healthtopics/study-quality-assessment-tools.

8. Zhang T, Cui X, ZhaoX, WangJ. Detectable SARS-CoV-2 viral RNA in feces of three children during recovery period of COVID-19 pneumonia. J MedVirol.2020;92:909-914.

9. Song W, Li J, Zou N, Guan W, Pan J, Xu W. Clinical features of pediatric patients with coronavirus disease (COVID-19). J ClinVirol. 2020 Jun; 127: 104377.

10. Tan Y, Tan B, Pan J, Wu J, Zeng S, Wei H. Epidemiologic and clinical characteristics of 10 children with coronavirus disease 2019 in Changsha, China. J ClinVirol. 2020 Jun; 127: 104353.

11. Cui Y, Tian M, Huang D, Wang X, Huang Y, Fan L, et al. A 55-DayOld Female Infant Infected With 2019 Novel Coronavirus Disease: Presenting With Pneumonia, Liver Injury, and Heart Damage. J Infect Dis 2020 May 11;221(11):1775-1781.

12. Lu Y, Wen H, Rong D, Zhou Z, Liu H. Clinical characteristics and radiological features of children infected with the 2019 novel coronavirus. ClinRadiol. 2020; 75:520-5.

13. Yin X, Dong L, ZhangY, Bian W, Li H. A mild type of childhood Covid-19 - A case report. Radiol Infect Dis 2020 Jun;7(2):78-80.

14. Chan JF, Yuan S, Kok KH, To KK, Chu H, Yang J, et al. A familial cluster of pneumonia associated with the 2019 novel coronavirus indicating person-to-person transmission: a study of a family cluster. Lancet. 2020 15-21 February; 395(10223): 514-23.

15. Xu R, Du M, Li L, Zhen Z, Wang H, Hu X. CT imaging of one extended family cluster of coronavirus disease 2019 (COVID-19) including adolescent patients and "silent infection." Quant Imaging Med Surg. 2020 Mar; 10(3): 800-4.

16. Salik I, Mehta B. Tetralogy of Fallot palliation in a COVID-19 positive neonate. J ClinAnesth. 2020 May 25: 109914.

17. Dallan C, Romano F, Siebert J, Politi S, Lacroix L, Sahyoun C. Septic shock presentation in adolescents with COVID-19.Lancet Child Adolesc Health 2020 Jul;4(7):e21-e23.
18. Dumpa V, Kamity R, Vinci AN,Noyola E, Noor A. Neonatal Coronavirus 2019 (COVID-19) Infection: A Case Report and Review of Literature. Cureus 12(5): e8165.

19. Musolino AM, Supino MC, Buonsenso D, Ferro F, Valentini P, Magistrelli A, et al. Lung Ultrasound in Children with COVID-19: Preliminary Findings. Ultrasound Med Biol. 2020 May; 46(8).

20. Verdoni L, Mazza A, Gervasoni A, Martelli L, Ruggeri M, Ciuffreda M, et al. An outbreak of severe Kawasaki-like disease at the Italian epicentre of the SARS-CoV-2 epidemic: an observational cohort study. Lancet 2020; 395: 1771-78

21. Park JY, Han MS, Park KU, Kim JY, Choi EH. First Pediatric Case of Coronavirus Disease 2019 in Korea. J Korean Med Sci. 2020 March 23;35(11):e124

22. Piersigilli F, Carkeek K, Hocq C, Grambezen BV, Hubinont C, Chatzis O, et al. COVID-19 in a 26 -week preterm neonate. Lancet Child Adolesc Health 2020 Jun; 4(6): 476-8.

23. Mansour A, Atoui R, Kanso K, Mohsen R, Fares Y, Fares J. First Case of an Infant with COVID-19 in the Middle East. Cureus. 2020 Apr; 12(4): e7520.

24. Patel NA. Pediatric COVID-19: Systematic review of literature. Am J Otolaryngol 2020; 41:102573.

25. Ludgvisson JF. Systematic review of COVID-19 in children shows milder cases and a better prognosis than adults. ActaPediatrica 2020; 00:1-8.

26. Yasahura J, Kuno T, Takagi H, Sumitomo N. Clinical characteristics of COVID-19 in children: A systematic review. PediatricPulmonology.2020;55:2565-2575.

27. Christophers B, Marin BG, Oliva R, Powell WT, Savage TJ, Michelow IC. Trends in clinical presentation of children with COVID-19: a systematic review of individual participant data. Pediatr Res (2020). https://doi.org/10.1038/s41390-020-01161-3

28. Brodin P. Why is COVID-19 so mild in children? ActaPaediatrica. 2020;109:1082-3.

29. American Academy of Pediatrics. Multisystem Inflammatory Syndrome in Children (MIS-C) Interim Guidance. [Internet]. [cited 2020 Nov 22]. Available from https://services.aap.org/en/ pages/2019-novel-coronavirus-covid-19-infections/clinical-guidance/ multisystem-inflammatory-syndrome-in-children-mis-c-interimguidance/

30. Parisi GF, Indolfi C, Decimo F, Leonardi S, del Giudeci MM. COVID-19 Pneumonia in Children: From Etiology to Management. Front Pediatr 2020 December 14;8:616622

31. Foust AM, Phillips GS, Chu WC, Daltro P, Das KM, Garcia-Peña $\mathrm{P}$, et al. International Expert Consensus Statement on Chest Imaging in Pediatric COVID-19 Patient Management: Imaging Findings, Imaging Study Reporting and Imaging Study Recommendations. Radiology Cardiothoracic Imaging 2020 April; 2(2):e200214

32. Nino G, Zember J, Sanchez-Jacob R, Gutierrez MJ, Sharma K, DPhil MG, Pediatric lung imaging features of COVID-19: A systematic review and meta-analysis. Pediatr Pulmonol 2021 Jan;56(1):252-63

33. Foust AM, Winant AJ, Chu WC, Das KM, Phillips GS, Lee EY. Pediatric SARS, H1N1, MERS, EVALI, and Now Coronavirus Disease (COVID-19) Pneumonia: What Radiologists Need to Know. AJR Am J Roentgenol 2020 Sep;215(3):736-44.

34. Kache S, Chisti MJ, Gumbo F, Mupere E, Zhi X, Nallasamy K, et al. COVID-19 PICU guidelines: for high and limited-resource settings. Pediatr Res 2020 Nov;88(5):705-16.

35. Pasnick S, Carlos WG, Dela Cruz CS; Gross JE, Garrison G, et al. Public Health Information Series: SARS-CoV-2 Transmission and the Risk of Aerosol-Generating Procedures. Am J Respir Crit Care Med 2020; 202:13-14.

36. Yonker LM, Neilan AM, Bartsch Y, Patel AB, Regan J, Arya P, et al. Pediatric Severe Acute Respiratory Syndrome Coronavirus 2 (SARSCoV-2): Clinical Presentation, Infectivity, and Immune Responses. J Pediatr 2020 Dec;227:45-52.e5. 